\title{
Prospective Controlled Animal Study on Biopsy Sampling with New Flexible Cryoprobes versus Forceps: Evaluation of Biopsy Size, Histological Quality and Bleeding Risk
}

\author{
Karl-Josef Franke ${ }^{a}$ Dirk Theegarten ${ }^{b}$ Claus Hann von Weyhern ${ }^{c}$ Georg Nilius ${ }^{a}$ \\ Christiane Brueckner $^{a} \quad$ Jürgen Hetzel ${ }^{d} \quad$ Martin Hetzel $^{f} \quad$ Karl-Heinz Ruhle $^{a}$ \\ Markus D. Enderle ${ }^{e}$ Mara N.I. Szyrach ${ }^{e}$ \\ ${ }^{a}$ Department of Pneumology and Critical Care Medicine, Helios Klinik Ambrock, University of Witten/Herdecke, \\ Hagen, ${ }^{b}$ Institute of Pathology and Neuropathology, University Hospital Essen, University Duisburg-Essen, \\ Essen, ' Institute of Pathology, University of Tübingen, d Department of Oncology, Hematology, Immunology, \\ Rheumatology and Pulmonology, University Hospital, Internal Medicine II, University of Tübingen, and \\ e ERBE Research, Tübingen, and ${ }^{\mathrm{f} C l i n i c s}$ for Pneumology and Internal Medicine, Red Cross Hospital, Stuttgart, \\ Germany
}

\section{Key Words}

Bleeding • Bronchoscopy · Cryobiopsy · Forceps biopsy

\begin{abstract}
Background: Cryoextraction is a procedure used for the recanalization of obstructed airways caused by visible exophytic endobronchial tumor. Biopsy samples obtained by this technique have been shown to be useful for histological assessment. Objectives: The aim of the present animal study was to systematically evaluate biopsy size, histological quality and bleeding risk after cryobiopsy with new, flexible cryoprobes in comparison with forceps biopsy, serving as the gold standard. Methods: Biopsies were obtained from anesthetized pigs with the flexible bronchoscopy technique, and evaluated histologically with respect to their size and quality. Bleeding frequency, bleeding duration and histological changes in the biopsy bed were also recorded. $R \boldsymbol{e}$ sults: Cryobiopsies were significantly larger than forceps bi-
\end{abstract}

opsies. The size of cryobiopsies was dependent on the freezing time. The histological quality of the cryobiopsy specimens was not impaired by the freezing process, whereas forceps biopsies showed typical crush artifacts. Despite the larger defects left in the tracheobronchial system after cryobiopsy, bleeding frequency and duration were not higher compared to forceps biopsy. Conclusions: Since cryobiopsy sampling is not associated with a higher bleeding risk compared with forceps biopsy, this new biopsy technique offers - in addition to a good specimen quality - a safe and valuable tool with the potential of improving the outcome of diagnostic endoscopy.

Copyright $\odot 2010$ S. Karger AG, Basel

Conflict of Interest: Markus D. Enderle is Medical Director and Head of ERBE Research, Germany, and Mara N.I. Szyrach is co-worker at ERBE Research, Germany.

\section{KARGER}

Fax +41613061234 E-Mail karger@karger.ch www.karger.com
(C) 2010 S. Karger AG, Base

$0025-7931 / 10 / 0802-0127 \$ 26.00 / 0$

Accessible online at:

www.karger.com/res
Karl-Josef Franke, MD

Department of Pneumology and Critical Care Medicine, Helios Klinik Ambrock

University of Witten/Herdecke, Ambrocker Weg 60, DE-58091 Hagen (Germany)

Tel. +49 2331974 2002, Fax +49 23319742009

E-Mail karl-josef.franke@helios-kliniken.de 


\section{Introduction}

In the central respiratory tract, cryotherapy is used for the treatment of early, superficial bronchial carcinomas [1] and for bronchoscopic recanalization of malignant and benign obstructions [2-5]. The cryoprobe tip is positioned on the tumor and several freezing and thawing cycles are initiated. On macroscopic inspection, the tissue appears to be undamaged. Within a period of $8-15$ days, however, the tissue becomes necrotic and has to be debrided in an additional bronchoscopic procedure $[4,6]$.

The development of new, flexible and mechanically more stable cryoprobes with a larger tip area allows the immediate removal of exophytic tumorous tissue from the bronchial system via the cryoadhesive effect [7]. The frozen tissue adheres to the probe's tip and is removed by withdrawing the probe together with the bronchoscope enabling piecemeal extraction of tissue. This feature allows the extraction of even freeze-resistant tissue such as fat in the same manner [8].

There is increasing evidence that the histopathological assessability of biopsies obtained with cryoprobes is not impaired by the freezing process - rather, the good quality of the biopsies has been demonstrated in clinical feasibility studies $[9,10]$.

A recent systematic ex vivo study [11] and initial clinical studies $[9,10]$ show that the tissue specimens extracted with this technique are larger than forceps biopsies. In the ex vivo study, it has been shown that the biopsy size depends on the parameters probe diameter, freezing duration, tissue type and the application pressure of the probe on the tissue [11]. To date, a systematic evaluation of these parameters in a perfused in vivo model is lacking.

Another important aspect of this new biopsy technique is its safety in terms of bleeding complications. The reported risk of bleeding from bronchoscopy with a blood loss of more than $20-50 \mathrm{ml}$ varies greatly $(0.12-$ $44.5 \%)$. The probability of a relevant bleeding is influenced by various factors, for example, a preceding lung transplantation or transbronchial biopsy [12-16]. The cytotoxic effect of tissue devitalization by cryotherapy is supposed to be largely due to the delayed formation of intravascular thrombosis leading to infarction [17]. Initial vasoconstriction within the cooled tissue is followed by vasodilatation with the formation of intravascular microthrombosis $[17,18]$. These mechanisms theoretically could be capable of reducing the risk of bleeding after cryobiopsy.
The primary aim of our animal study was to confirm our ex vivo findings [11] in an in vivo animal setting, i.e. to evaluate the size and histological quality of cryobiopsy samples in dependency of the diameter of the tip of the cryoprobe and the freezing period, and to compare the results with those of forceps biopsy. The secondary aim was to evaluate the bleeding frequency and duration as well as to compare the histological appearance of the resulting tissue lesion at the extraction site (biopsy bed).

\section{Materials and Methods}

\section{Animal Model}

Biopsy sampling was performed in 3 Duroc/Pietrain hybrid pigs weighing $120 \pm 5 \mathrm{~kg}$. The animals were premedicated with ketamine $(90 \mathrm{mg} / \mathrm{kg}$ body weight), xylazine $(10 \mathrm{mg} / \mathrm{kg}$ body weight) and atropine. Anesthesia was induced with fentanyl, midazolam and pancuronium and maintained with isoflurane/fentanyl after intubation. Normal saline was infused intravenously at $9 \mathrm{ml} / \mathrm{kg} / \mathrm{h}$ to maintain homeostasis. After tracheotomy, the animals were intubated with a 9.0-mm Bronchoflex tube (Teleflex Medical GmbH, Kernen, Germany). Biopsies were extracted with cryoprobes or forceps inserted via a bronchoscope (FB-18P, Pentax Europe, working channel $2.8 \mathrm{~mm}$ ) to mimic the clinical situation.

All experiments were performed in accordance with the federal law regarding the protection of animals. The principles of laboratory animal care (NIH Publication No. 85-23, revised 1985) were complied with. The study was approved by the governmental review board.

\section{Equipment and Experimental Setting}

Mucosal biopsies were obtained with 1.9- and 2.4-mm cryoprobes (fig. 1) (ERBE, Tübingen, Germany) activated for different, clinically relevant periods $(1,2$, and $3 \mathrm{~s})$ or with forceps biopsy. The extraction sites for the different instruments or activation times were randomly distributed in the airway and, this again, varied from animal to animal.

The cooling principle of the probe is based on the JouleThompson effect by means of a sudden decompression of a compressed gas that results in a cooling effect at the probe's tip. The cooling agent is applied under high pressure through the central canal of the probe (Erbokryo CA, ERBE Tübingen, Germany). Carbon dioxide was used as coolant gas.

For comparison, biopsies were taken with biopsy forceps (diameter $2.4 \mathrm{~mm}$, Pentax Europe). With each parameter setting (different cryoprobe tip diameters and freezing times or forceps) 5 biopsies were taken per animal, giving a total of 35 biopsies per pig. Biopsies were extracted from the distal trachea, the main bronchi, and the proximal upper and lower lobe bronchi. Bleeding complications were noted by recording whether a bleeding occurred or not, and by measuring the bleeding duration from the time of sampling to cessation of bleeding, i.e. until no more fresh blood was visible after suctioning in addition to a clearly visible biopsy bed. 


\section{Sample Processing and Histology}

The weight of the biopsies was determined with a precision balance (Shimadzu AW 120, A. Schumann GmbH, Sillerup, Germany) immediately after biopsy sampling. The weight of the fluid remaining on the balance after weighing the sample was subtracted from the overall weight before transferring the samples into $4.0 \%$ buffered formaldehyde (Rotifix, Roth, Karlsruhe, Germany). The mean diameter of the biopsies was measured in two dimensions per sample in a nonblinded fashion (D.T.). Afterwards the biopsies were embedded in paraffin (Paraplast, McCormick Scientific, St. Louis, Mo., USA), cut into $5-\mu \mathrm{m}$ cross sections and stained with hematoxylin (Fluka, Steinheim, Germany) and eosin (Waldeck, Münster, Germany). The relative artifact-free area was determined by a second pathologist (C.H.W.) blinded to the biopsy technique.

The trachea and bronchi were sectioned, beginning from the central area, and the sections were embedded in paraffin and stained in the usual manner. Analysis of the tissue changes at the biopsy site was performed under a light microscope (D.T.) (Nikon Eclipse E800, Japan).

\section{Statistics}

Data were analyzed by means of descriptive statistics (means and standard deviations) as well as by statistical hypothesis testing and linear regression analysis. For independent samples, Student's t test was used to compare normally distributed variables among the groups. To account for inhomogeneity of the variations between the groups, Welch's correction was used. For nonnormally distributed samples, a Mann-Whitney test was used. Pearson's regression analysis was performed to determine the correlation between biopsy weight and diameter versus the freezing period. All $\mathrm{p}$ values $(\mathrm{p}<0.05$ was considered statistically significant) are two-sided and were not adjusted for the number of parameters evaluated.

\section{Results}

\section{Cryobiopsies Extracted from Perfused Tissue Are}

Larger than Forceps Biopsies

With the 2.4-mm cryoprobe the weight of the samples was significantly higher than that of forceps biopsies for all activation periods ( $\mathrm{p}<0.0001)$, and with the $1.9-\mathrm{mm}$ cryoprobe after freezing periods of 2 and $3 \mathrm{~s}(\mathrm{p}=0.0007$ and $\mathrm{p}=0.0001$, respectively) (fig. 2). Concerning the freezing time dependency, a significant increase in biopsy weight was observed after $3 \mathrm{~s}$ with the $1.9-\mathrm{mm}$ cryoprobe $(\mathrm{p}=0.0047)$ compared to a freezing time of $2 \mathrm{~s}$, and after $2 \mathrm{~s}$ with the $2.4-\mathrm{mm}$ probe $(\mathrm{p}=0.0002)$ compared to a freezing time of $1 \mathrm{~s}$ (fig. 2). For all activation periods, only a moderate correlation between freezing period and biopsy weight could be demonstrated $(\mathrm{r}=0.54,2.4-\mathrm{mm}$ probe; $r=0.50,1.9-\mathrm{mm}$ probe).

The diameter of cryobiopsies was at least 30\% larger than that of forceps biopsies (table 1). Significantly larger

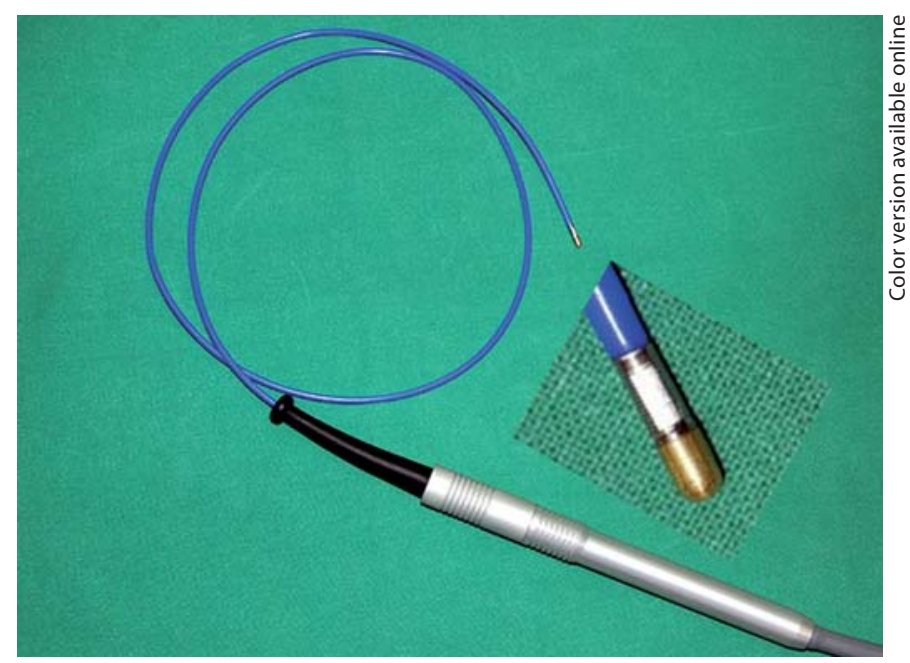

Fig. 1. Flexible cryoprobe, $2.4 \mathrm{~mm}$ in diameter.

Table 1. Biopsy diameter in dependency of the instrument and freezing duration

\begin{tabular}{|c|c|c|c|c|c|c|c|}
\hline & \multirow{2}{*}{$\begin{array}{l}\text { For- } \\
\text { ceps }\end{array}$} & \multicolumn{3}{|c|}{ 1.9-mm cryoprobe } & \multicolumn{3}{|c|}{ 2.4-mm cryoprobe } \\
\hline & & $1 \mathrm{~s}$ & $2 \mathrm{~s}$ & $3 \mathrm{~s}$ & $1 \mathrm{~s}$ & $2 \mathrm{~s}$ & $3 \mathrm{~s}$ \\
\hline \multicolumn{8}{|l|}{ Mean diameter } \\
\hline $\mathrm{mm}$ & 2.4 & 4.3 & 3.6 & 4.5 & 4.0 & 5.2 & 5.1 \\
\hline SD & 1.2 & 2.9 & 3.0 & 4.3 & 2.8 & 4.2 & 4.0 \\
\hline
\end{tabular}

diameters were observed after $1 \mathrm{~s}$ for the 1.9-mm cryoprobe $(\mathrm{p}=0.0081)$ and after all activation times for the $2.4-\mathrm{mm}$ cryoprobe $(\mathrm{p}=0.0014$ after $1 \mathrm{~s}, \mathrm{p}=0.0027$ after $2 \mathrm{~s}$, and $\mathrm{p}=0.0002$ after $3 \mathrm{~s}$ ) compared to forceps.

\section{Cryobiopsies Are Structurally Intact, and Tissue}

Integrity Is Not Impaired by the Freezing Process

Histologically, cryobiopsies as well as forceps biopsies were of excellent quality for microscopic evaluation. The artifact-free area was $>95 \%$ for both, forceps and cryobiopsy samples. Only in very rare cases was the artifact-free area lower: in 2 samples taken with the $2.4-\mathrm{mm}$ cryoprobe after a 1-second freezing time and in 1 case after a freezing period of $3 \mathrm{~s}$. Artifacts comprised slight crush artifacts in forceps biopsies, fibrin deposits at the margins of cryobiopsies and slight tissue fragmentation due to sample processing (i.e. cutting and slide preparation). Cryospecific artifacts were not observed. 


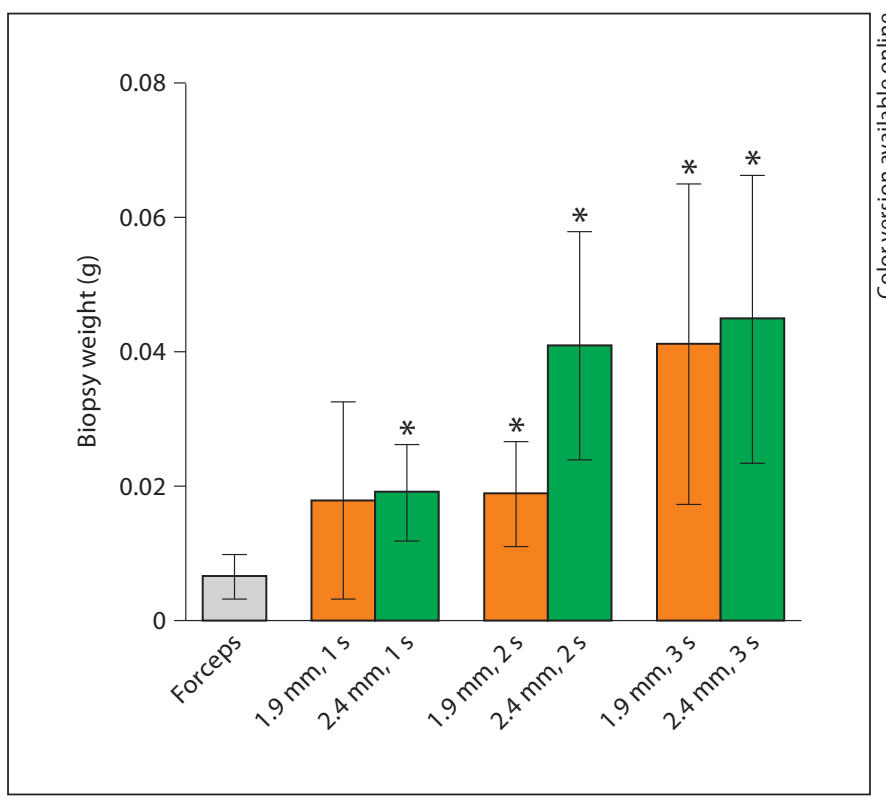

Fig. 2. Biopsy weight in dependency of the biopsy instrument and freezing duration (mean $\pm S D)$. Significantly $(p<0.05)$ heavier cryobiopsies compared to forceps biopsies are marked with asterisks.

Despite the Larger Dimensions of the Samples,

Cryobiopsy Is Not Associated with More Bleeding than Forceps Biopsy

Minimal oozing bleeding - treated by suction - occurred after any biopsy, irrespective of the biopsy technique applied. No severe bleeding requiring an intervention was observed. Bleeding intensity was comparable and minimal (below $20 \mathrm{ml}$ ) independent of the biopsy instrument or the activation times used. There was no significant difference in bleeding times after biopsy sampling with cryoprobe or forceps ( $p>0.1$ ), and no difference was observed between the two cryoprobes at the different freezing periods ( $\mathrm{p}>0.1$ ) (fig. 3).

\section{The Histological Appearance of the Biopsy Bed Is}

Similar after Cryobiopsy and Forceps Biopsy

No qualitative difference was seen between cryobiopsy and forceps biopsy beds. Under the microscope, artifacts corresponding to those observed in the biopsy specimens were also seen in the biopsy bed, which was covered with fibrin lamellae of varying size. Discrete bleeding into the periphery and focal edema was seen. Thrombus formation within the capillaries was not found.

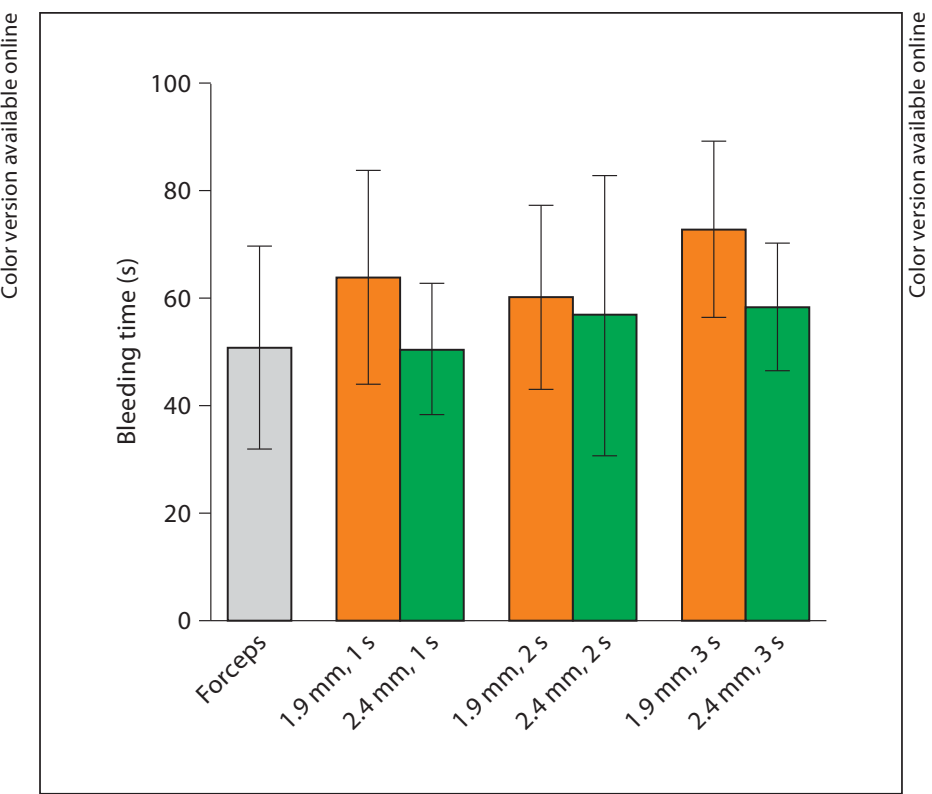

Fig. 3. Bleeding time in dependency of the biopsy instrument and freezing period (mean $\pm \mathrm{SD}$ ).

\section{Discussion}

In an in vivo animal model, we could confirm our prior ex vivo findings and thereby demonstrate that, even under perfused conditions, it is possible to obtain significantly larger biopsies with cryoprobes than with forceps. Additionally, bleeding duration after biopsy was comparable with both biopsy techniques despite the larger specimens acquired with the cryoprobes. The biopsy beds showed no qualitative differences.

The possibility of extracting tissue from the bronchial system with the cryoadhesive effect between the probe tip and tissue led to the idea that the extracted tissue might be useful for histopathological examination. Bonniot et al. [19] showed that the histopathological assessability of forceps biopsy samples does not seem to be impaired by the freezing process when biopsies are obtained immediately after cryotherapy. In a retrospective study in 12 patients suffering from exophytic endobronchial malignancies, cryobiopsies were found to be extremely good in terms of size and quality [9]. The median diameter of biopsies obtained with the $2.4-\mathrm{mm}$ cryoprobe was $6.7 \mathrm{~mm}$. In our experiments, the mean diameter of samples taken with the $2.4-\mathrm{mm}$ probe was 5.2 and $5.1 \mathrm{~mm}$ after 2 - and 3 -second activation periods, respectively. The weight and 
diameter of cryobiopsies were 60 and $30 \%$ higher than those of forceps biopsies for both cryoprobes used, even after the shortest freezing period of $1 \mathrm{~s}$ (fig. 2; table 1). These data are in agreement with the results of an ex vivo animal study in which cryobiopsies taken with the 2.4$\mathrm{mm}$ cryoprobe at an activation of $1 \mathrm{~s}$ were almost twice as heavy as specimens obtained by forceps biopsy, and the diameter 29\% (1.9-mm cryoprobe) and 56\% (2.4-mm cryoprobe) larger than that of forceps biopsies [11]. In another feasibility study involving 41 patients, transbronchial cryobiopsies were performed for the diagnosis of diffuse lung disease [10]. In this study, cryobiopsies were $60 \%$ larger than the forceps biopsies. The variability between these studies can be explained by the different types of tissue biopsied, and by variable or nonexistent (ex vivo study) tissue perfusion. In contrast to biopsies taken from porcine, nonperfused lung tissue, we observed little or no correlation between biopsy weight or diameter and freezing period. This is most probably due to the fact that the tissue perfusion tends to inhibit the freezing process [20] and also because of the different freezing capacities of the cryoprobes due to their different tip diameters. Consequently, a significant increase in biopsy weight was only achieved after $2 \mathrm{~s}$ with the $1.9-\mathrm{mm}$ cryoprobe, whereas a significant increase was already visible after $1 \mathrm{~s}$ with the 2.4-mm cryoprobe. Another explanation is the limited depth of the mucosa restricting the biopsy size. This fact also accounts for the extraction of cartilage in rare cases. In addition, in contrast to the ex vivo study with the cryoprobe placed perpendicularly on the tissue [11], we extracted the biopsies with the cryoprobe inserted through a bronchoscope. Since perpendicular placement of the cryoprobe was not always feasible, a more or less lateral adhesion of tissue to the probe was observed. This difference also explains the considerable variability in biopsy diameter (table 1). However, it is just this possibility of extracting biopsies with the cryoprobe tip applied laterally that represents a clear advantage, as it allows biopsy sampling from areas that are difficult or impossible to access with biopsy forceps.

Regarding the histological quality of cryobiopsies, Hetzel et al. [9] observed that the artifact-free area was better than $75 \%$ in 9 out of 12 patients, and that molecular markers were well preserved, enabling the establishment of an immunohistochemical diagnostics. Forceps biopsies, in contrast, often exhibit crush artifacts, making a diagnosis difficult or even impossible, especially in small biopsy samples. In our study, the artifact-free area was $>95 \%$ for both forceps and cryobiopsy samples. We also observed typical crush artifacts in forceps biopsies and, in rare case, cryobiopsies revealed small foci of fibrin exudation which did not impair the diagnostic procedure.

A possible explanation for the good quality of forceps biopsies in this study might be the fact that healthy mucosa is less sensitive to mechanical or thermal influences than tumor or parenchymal tissue. It must be emphasized that longer activation periods did not influence histological quality, since the area containing artifacts was comparable over all freezing periods (1,2 and $3 \mathrm{~s})$. Nevertheless, although the artifact-free area was $>95 \%$ for both, forceps and cryobiopsies, the absolute size of the artifact-free area is larger in cryobiopsies due to their overall larger size and thus may represent an advantage for the pathologist to establish a definite diagnosis.

Extracting large biopsies might appear likely to cause more profuse bleeding, but this assumption was not confirmed in our study. Bleeding frequency and duration were similar with both forceps and cryoprobes (and independent of cryoprobe diameter and freezing period) despite the fact that cryobiopsies were significantly larger (fig. 2). Cold-induced vascular microthrombosis was not demonstrable. The vasoconstrictory effect may therefore account for the short bleeding duration despite the larger defects created at the site of tissue sampling $[17,18]$.

In summary, the superiority of cryobiopsies over forceps biopsies will be determined clinically when this technique leads to a significantly improved diagnostic yield with the same or a lower complication rate. Although the results of the present study were obtained in an animal model, it may be expected that they can be repeated in the clinical setting in humans. These assumptions are the subject of an ongoing prospective, controlled and randomized multicenter study.

Although the larger samples obtained by cryobiopsy improve diagnostic quality, they do have one disadvantage: currently, the tissue sample adhering to the cryoprobe cannot be withdrawn through the working channel of the endoscope, but must be removed together with the endoscope. The possibility of recovering the biopsy together with the cryoprobe through the working channel of the endoscope would be a desirable future development and is currently in process. 


\section{References}

$\checkmark 1$ Deygas N, Froudarakis M, Ozenne G, Vergnon JM: Cryotherapy in early superficial bronchogenic carcinoma. Chest 2001;120: 26-31.

-2 Asimakopoulos G, Beeson J, Evans J, Maiwand MO: Cryosurgery for malignant endobronchial tumors: analysis of outcome. Chest 2005;127:2007-2014.

-3 Maiwand MO, Asimakopoulos G: Cryosurgery for lung cancer: clinical results and technical aspects. Technol Cancer Res Treat 2004;3:143-150.

4 Mathur PN, Wolf KM, Busk MF, Briete WM, Datzman M: Fiberoptic bronchoscopic cryotherapy in the management of tracheobronchial obstruction. Chest 1996;110:718-723.

$\checkmark 5$ Sheski FD, Mathur PN: Cryotherapy, electrocautery, and brachytherapy. Clin Chest Med 1999;20:123-138.

-6 Vergnon JM, Huber RM, Moghissi K: Place of cryotherapy, brachytherapy and photodynamic therapy in therapeutic bronchoscopy of lung cancers. Eur Respir J 2006;28:200218.

7 Hetzel M, Hetzel J, Schumann C, Marx N, Babiak A: Cryorecanalization: a new approach for the immediate management of acute airway obstruction. J Thorac Cardiovasc Surg 2004;127:1427-1431.
8 Franke KJ, Nilius G, Ruhle KH: Cryorecanalization of an endobronchial lipoma. Pneumologie 2005;59:685-688.

$\checkmark 9$ Hetzel J, Hetzel M, Hasel C, Moeller P, Babiak A: Old meets modern: the use of traditional cryoprobes in the age of molecular biology. Respiration 2008;76:193-197.

10 Babiak A, Hetzel J, Krishna G, Fritz P, Moeller P, Balli T, Hetzel M: Transbronchial cryobiopsy: a new tool for lung biopsies. Respiration 2009;78:203-208.

11 Franke KJ, Szyrach M, Nilius G, Hetzel J, Hetzel M, Ruehle KH, Enderle MD: Experimental study on biopsy sampling using new flexible cryoprobes: influence of activation time, probe size, tissue consistency, and contact pressure of the probe on the size of the biopsy specimen. Lung 2009;187:253-259.

12 Pue CA, Pacht ER: Complications of fiberoptic bronchoscopy at a university hospital. Chest 1995; 107:430-432.

13 Bjortuft O, Brosstad F, Boe J: Bronchoscopy with transbronchial biopsies: measurement of bleeding volume and evaluation of the predictive value of coagulation tests. Eur Respir J 1998;12:1025-1027.

14 Diette GB, Wiener CM, White P Jr: The higher risk of bleeding in lung transplant recipients from bronchoscopy is independent of traditional bleeding risks: results of a prospective cohort study. Chest 1999;115:397402.
15 Milman N: Diffuse bleeding from the bronchial mucosa on flexible bronchoscopy in lung transplanted patients: 'the hemorrhagic bronchial mucosa syndrome'. Respiration 2009;77:416-419.

16 Diaz-Guzman E, Vadi S, Minai OA, Gildea TR, Mehta AC: Safety of diagnostic bronchoscopy in patients with pulmonary hypertension. Respiration 2009;77:292-297.

17 Homasson JP: Bronchoscopic cryotherapy. J Bronchol 1995;2:145-153.

18 Vergnon JM, Mathur PN: Cryotherapy for endobronchial disorders; in Bolliger CT, Mathur PN (eds): Interventional Bronchoscopy. Progress in Respiratory Research. Basel, Karger, 2000, vol 30, pp 133-145.

19 Bonniot JP, Homasson JP, Roden SL, Angebault ML, Renault PC: Pleural and lung cryobiopsies during thoracoscopy. Chest 1989; 95:492-493.

20 Jungraithmayr W, Szarzynski M, Neeff H, Haberstroh J, Kirste G, Schmitt-Graeff A, Farthmann EH, Eggstein S: Significance of total vascular exclusion for hepatic cryotherapy: an experimental study. J Surg Res 2004; 116:32-41. 